\title{
Sparse Chirp Stepped-frequency ISAR Super-resolution Imaging Method Based on 2D-FISTA Algorithm
}

\author{
Ming Zhou, Peng-Cheng Xu, Zhao-Hui He \\ Air Force Early Warning Academy, \\ Wuhan, 430019, China \\ E-mail: yasha1234@163.com, 40771912@qq.com
}

\author{
Qing-Hua You \\ Unit 95980 of the Chinese People Liberation Army, \\ Xiangyang, 441000, China \\ E-mail: 445479018@qq.com
}

\begin{abstract}
The traditional Sparse Chirp Stepped-frequency ISAR imaging method has the problem of low imaging quality and large computational complexity. In view of the above problems, this paper studies a sparse Chirp Stepped-frequency ISAR imaging method based on two-dimensional fast iterative shrinkage threshold algorithm (2D-FISTA). Firstly, the two-dimensional echo model of Sparse Chirp Stepped-frequency signal is constructed. Based on this model, two-dimensional fast iterative shrinkage threshold algorithm is used to reconstruct the imaging result. This method can be reconstructed in the matrix domain directly, which avoids the computation of the vector processing method, and avoids the problem of the image quality loss of the decoupling method. The simulation results show that the proposed method is effective and has better imaging performance.
\end{abstract}

Keywords-sparse chirp stepped-frequency signal; 2D-FISTA; compressive sensing; ISAR super-resolution imaging

\section{INTRODUCTION}

Synthetic Aperture Radar /Inverse Synthetic Aperture Radar (SAR/ISAR) is mainly to achieve high range resolution by increasing the signal bandwidth. Stepped-frequency Chirp Signal (SFCS) by transmitting a set of continuous linear frequency carrier frequency hopping realization synthetic large bandwidth. It has the advantages of low system complexity, low cost, easy to realize and so on, which has become the development trend of high resolution radar technology $[1,2,3]$. Because of the number of sub pulses transmitted by the SFCS, the imaging time is long. Therefore, in practice, the echo signal is usually sparse. The traditional processing method in dealing with the Sparse Stepped-frequency Chirp Signal (SSFCS) will appear when the performance degradation or even invalidate, increases the difficulty of signal processing. In recent years, with the theory of compressive sensing [4] has been widely used in SAR/ISAR imaging system, it provides a new way to solve the problem of sparse signal imaging $[5,6,7]$.

In $[8,9,10,12]$, the matrix is transformed into vector form firstly, and then the sparse signal imaging is carried out. The above research is based on one dimensional reconstruction algorithm or the row and column stacking method to reconstruct, so the computation is large. Literature [13] uses MSL0 algorithm to reconstruct the distance direction and azimuth direction, which can further reduce the computational complexity. However, this method did not consider the distance and azimuth coupling information, so the image quality degradation. A 2D-OMP algorithm is proposed in [14], but it is necessary to set up a specific set of sparse dictionary, so it is not widely used. The concept of two-dimensional random projection is proposed in [15], and the idea of using 2D-SL0 to reconstruct the random projection is analyzed. Literature $[16,17,18]$ proposed $2 \mathrm{D}$ iterative adaptive method for 2D signal reconstruction, and obtain a better reconstruction effect, but the method has higher computational complexity too.

\section{SSFCS ECHO MODEL}

A set of SFCS can be expressed as [12]:

$$
S(t)=\sum_{n=0}^{N-1} \operatorname{rect}\left[\frac{t-n T_{r}}{T}\right] \exp \left[j \pi \mu\left(t-n T_{r}\right)^{2}\right] \exp \left(j 2 \pi f_{n}\left(t-n T_{r}\right)\right)
$$

Where $\operatorname{rect}(\bullet) \quad$ is a window function, $f_{n}=f_{0}+n \Delta f, n=0,1,2 \ldots \ldots . N-1, N$ is sub-pulse number, $f_{0}$ is carrier frequency, $t$ is the time, $T$ is sub-pulse width, $\Delta f$ is carrier frequency step and the equivalent signal bandwidth is $B=N \Delta f$.

Assuming that the target's motion compensation has been completed and transformed into an equivalent table model, the detailed motion compensation method can be referenced in [1]. Then, the sampling signal obtained by the sub-pulse compression processing which is reflected by the $I$ scattering points and the distance from radar is $R_{i}\left(t, t_{m}\right)$.

$$
U_{n, m}=\sum_{i=1}^{I} \sigma_{i} \exp \left[-j 4 \pi\left(f_{0}+n \Delta f\right) \frac{R_{i}\left(t, t_{m}\right)}{c}\right]
$$

For the target turntable model, the distance between the $i(i=1, \ldots . . . I)$ scattering point and the radar at the ${ }^{t}$ time can be expressed as [12]: 


$$
R_{i}\left(t, t_{m}\right) \approx R_{0}+x_{i} \cos \left[\theta\left(t_{m}\right)\right]-y_{i} \sin \left[\theta\left(t_{m}\right)\right]
$$

Where, $R_{0}$ is Initial distance, $x_{i}, y_{i}$ are the horizontal and vertical coordinates of the ${ }^{i}$ scattering point on the target coordinate system, $\theta\left(t_{m}\right)$ is the rotation angle of the target related to the slow time $t_{m}$. When the target rotation angle is usually relatively small during the observation time, $\cos \left(\theta\left(t_{m}\right)\right) \approx 1 \sin \left(\theta\left(t_{m}\right)\right) \approx \theta\left(t_{m}\right)$. Therefore, the formula (3) constant is incorporated into the amplitude information and is brought into the Eq. 2:

$$
\left.U_{n, m}=\sum_{i=1}^{I} \sigma_{i} \exp \left(-j 2 \pi f_{0} \frac{2 x_{i}}{c}\right) \exp \left(-j 2 \pi n \Delta f \frac{2 x_{i}}{c}\right) \exp \left[j 2 \pi\left(f_{0}+n \Delta f\right) 2 y_{i} m \Delta \theta / c\right)\right]
$$

Because the signal in range direction and azimuth sampling points were $N$ and $M$, so the distance resolution of is $\Delta x=c /(2 N \Delta f)$ and azimuth resolution is $\Delta y=\lambda_{0} /(2 M \Delta \theta)$, where $\lambda_{0}=c / f_{o}$. According to the distance and azimuth resolution, the target is discretized and the discrete form of the target area $\sigma=\left[\sigma_{p q}\right]_{P \times Q}$ is obtained. Where, $P=N J, Q=M J, J$ is the range and azimuth super resolution ratio. Because $\lambda_{n} \approx \lambda_{0}$, so Eq. 4 can be written as:

$$
U_{n, m}=\sum_{q=0}^{M-1} \sum_{p=0}^{N-1} \sigma_{p q} \exp \left(-j 2 \pi \frac{p n}{P}\right) \exp \left(j 2 \pi \frac{q m}{Q}\right)
$$

The Eq. 5 is written in matrix form:

$$
\mathrm{U}=\psi_{\mathrm{r}} \sigma \psi_{\mathrm{a}}^{T}
$$

Where,

$$
\begin{aligned}
& \mathbf{U}=\left[U_{n, m}\right]_{N \times M}, \quad \boldsymbol{\sigma}=\left[\sigma_{p q}\right]_{N \times M}, \quad \boldsymbol{\Psi}_{\mathbf{r}}=\left[\exp \left(-j 2 \pi \frac{p n}{P}\right)\right]_{N \times N}, \\
& \boldsymbol{\psi}_{\mathbf{a}}=\left[\exp \left(j 2 \pi \frac{q m}{Q}\right)\right]_{M \times M}
\end{aligned}
$$

When the distance and azimuth are sparse, the SSFCS echo model can be written as:

$$
\boldsymbol{Y}=\boldsymbol{D}_{\mathrm{r}} \psi_{\mathrm{r}} \boldsymbol{\sigma} \psi_{\mathrm{a}}^{\mathrm{T}} \boldsymbol{D}_{\mathrm{a}}^{\mathrm{T}}
$$

Among them, $\boldsymbol{Y}$ is sparse echo data matrix, $\mathbf{D}_{\mathrm{r}}$ is distance to sparse matrix, $\mathbf{D}_{\mathrm{a}}$ is azimuth sparse matrix.
Obviously, the above Eq. 7 is an ill conditioned equation, and there are infinitely many solutions. The optimal solution can be obtained by using the theory of compressed sensing.

\section{SSFCS-ISAR JOINT IMAGING METHOD}

According to CS theory, if the target is sparse or compressible, it can accurately reconstruct the target and the observed value can be significantly reduced by compressed sensing algorithm [4]. In fact, when the resolution is determined, the target can only contain a finite number of scattered points, that is, the object is sparse and can be reconstructed by using compressed sensing theory.

Assuming $A=D_{\mathrm{r}} \psi_{\mathrm{r}}, B=\psi_{\mathrm{a}} D_{\mathrm{a}}$, the Eq. 7 can be written:

$$
\mathbf{Y}=\boldsymbol{A} \boldsymbol{G} \boldsymbol{B}^{T}
$$

Under the condition of complex noise, the Eq. 8 can be converted to the unconstrained optimization problem.

$$
\hat{G}=\arg \min _{X \in C^{X \times M}} \frac{1}{2}\left[r^{\prime}\left[\left(Y-A G B^{I}\right)^{H}\left(Y-A G B^{I}\right)\right]+\lambda\|G\|_{1}\right.
$$

Where, $\lambda$ is the regularization parameter, which is used to control the balance between the precision of estimation and the sparse of the row. Eq. 9 can be quickly solved by FISTA theory [19].FISTA optimization algorithm is proposed based on Nestrov algorithm, its convergence rate is two times $O\left(1 / k^{2}\right)$. It has the same order of convergence rate as the optimal first order optimization algorithm. Therefore, it has the advantage of fast convergence.

Assuming that the auxiliary variable of the first $k$ iteration is $\boldsymbol{Z}^{k}$, and $\boldsymbol{G}^{k}$ for the reconstruction result of $k$ iterations obtained, then $\boldsymbol{Z}^{k}$ update formula is:

$$
\boldsymbol{Z}^{k}=\boldsymbol{G}^{k}+\frac{t_{k-1}-1}{t_{k}}\left(\boldsymbol{G}^{k}-\boldsymbol{G}^{k-1}\right)
$$

$$
\begin{aligned}
& \text { Assuming } \\
& F(\boldsymbol{G})=\operatorname{tr}\left[\left(\boldsymbol{A} \boldsymbol{G} \boldsymbol{B}^{T}-\boldsymbol{Y}\right)^{H}\left(\boldsymbol{A} \boldsymbol{G} \boldsymbol{B}^{T}-\boldsymbol{Y}\right)\right]+\mu\|\boldsymbol{G}\|_{1} \text {, then the } \\
& \text { two approximation function of } F(\boldsymbol{G}) \text { in the auxiliary } \\
& Q_{L}\left(\boldsymbol{G}, \boldsymbol{Z}^{k}\right)=\operatorname{tr}\left[\left(\boldsymbol{A} \boldsymbol{Z}^{k} \boldsymbol{B}^{T}-\boldsymbol{Y}\right)^{H}\left(\boldsymbol{A} \boldsymbol{Z}^{k} \boldsymbol{B}^{T}-\boldsymbol{Y}\right)\right]+\left\langle\boldsymbol{G}-\boldsymbol{Z}^{k}, \nabla\left\{\boldsymbol{Z}^{k}\right\}\right\rangle \\
& +\frac{L}{2} \operatorname{tr}\left(\left(\boldsymbol{G}-\boldsymbol{Z}^{k}\right)^{H}\left(\boldsymbol{G}-\boldsymbol{Z}^{k}\right)\right)+\mu\|\boldsymbol{G}\|_{1}
\end{aligned}
$$


Where, $\nabla\left\{\boldsymbol{Z}^{k}\right\}$ is gradient vector, its value is:

$$
\nabla\left\{\boldsymbol{Z}^{k}\right\}=\frac{\partial\left\{t r\left[\left(\boldsymbol{A} \boldsymbol{Z}^{k} \boldsymbol{B}^{T}-\boldsymbol{Y}\right)^{H}\left(\boldsymbol{A} \boldsymbol{Z}^{k} \boldsymbol{B}^{T}-\boldsymbol{Y}\right)\right]\right\}}{\partial \boldsymbol{Z}^{k}}=2 \boldsymbol{A}^{H}\left(\boldsymbol{A} \boldsymbol{Z}^{k} \boldsymbol{B}^{T}-\boldsymbol{Y}\right) \boldsymbol{B}^{*}
$$

Eq. 11 has a unique minimal solution. For the convenience of using iterative soft threshold algorithm for solving algebraic approximation, transformation of $Q_{L}\left(\boldsymbol{G}, \boldsymbol{Z}^{k}\right)$, while ignoring the constant term can be obtained:

$$
P_{L}\left(Z^{k}\right)=\underset{G}{\arg \min }\left\{\frac{L}{2}\left\|G-\left(Z^{k}-\frac{1}{L} A^{H}\left(A Z^{k} B^{T}-Y\right) B^{*}\right)\right\|_{F}^{2}+\mu\|G\|_{1}\right\}
$$

Where, $P_{L}\left(\boldsymbol{Z}^{k}\right)$ is the optimal solution.

Since $\|\boldsymbol{G}\|_{1}$ is a separable variable, the Eq. 13 can be solved by using soft threshold function, and the update formula for $G^{k}$ is obtained:

$$
\boldsymbol{G}^{k+1}=P_{L}\left(\boldsymbol{Z}^{k}\right)=\operatorname{csoft}\left(\boldsymbol{Z}^{k}-\frac{1}{L} \nabla\left\{\boldsymbol{Z}^{k}\right\}, \frac{\mu}{L}\right)
$$

Note that in the Eq. 13, $\boldsymbol{A}^{H} \boldsymbol{A}, \boldsymbol{B}^{\boldsymbol{T}} \boldsymbol{B}^{*}$ and $\boldsymbol{A}^{H} \boldsymbol{Y} \boldsymbol{B}^{*}$ are constants, can be computed in the iterative initialization, which can save computation. Finally summed up the flow chart of 2D-FISTA are shown in the following table.

TABLE I 2D-FISTA ALGORITHM

2D-FISTA algorithm:

Input: $\boldsymbol{A}, \boldsymbol{B}, \boldsymbol{Y}$
Initialization: $\quad L=L(f) \quad, \quad \boldsymbol{X}^{0}=\mathbf{0}$
$\mu=\zeta\left\|\boldsymbol{A}^{H} \boldsymbol{Y} \boldsymbol{B}^{*}\right\|_{\infty}$
$\quad$ Step ${ }^{k}$ iterative process:
First step: Calculate $\boldsymbol{Z}^{k} \quad$ and its gradient
matrix $\nabla\left\{\boldsymbol{Z}^{k}\right\}$.
$\quad$ Second $\quad$ step: $\quad \boldsymbol{G}^{k+1}$
$\boldsymbol{G}^{k+1}=\operatorname{csoft}\left(\boldsymbol{Z}^{k}-\frac{1}{L} \nabla\left\{\boldsymbol{Z}^{k}\right\}, \frac{\mu}{L}\right)$
Third step: Update step size $t_{k+1}: t_{k+1}=\sqrt{1+4 t_{k}^{2}} / 2$
Output: After the stop condition is satisfied, the final
iteration result is obtained $\hat{\boldsymbol{G}}=\boldsymbol{G}^{k}$.

In order to further reduce the number of iterations of the FISTA algorithm, we can take the idea of changing ${ }^{\mu}$, that is $\mu^{k+1}=\beta \mu^{k} . \beta$ is attenuation factor. In the initial iteration, to support range of sparse signal accurately determined, so we should choose a large $\beta$, general $0.6<\beta<1$. When the iterative error $\left\|\boldsymbol{Y}-\boldsymbol{A} \boldsymbol{G} \boldsymbol{B}^{T}\right\|_{F}^{2}$ reaches the threshold $\beta$, it shows that the sparse degree of the signal is close to the demand. At this time, it is needed to speed up the convergence rate. Therefore, choosing $0<\beta<0.5$ can further improve the convergence rate.

\section{SIMULATION ANALYSIS AND VERIFICATION}

Assuming that the simulation parameters are set as follows: $M=512, N=256, f_{0}=10 \mathrm{GHz}, T=5 \mu s, \Delta f=2 \mathrm{MHz}$, $B=2 \mathrm{MHz}, \mathrm{PRF}=200 \mathrm{~Hz}, R_{0}=50.5 \mathrm{Km}, \mathrm{SNR}=10 \mathrm{~dB}$, $J=3$. The target model is shown in Figure 1, the angle between the target flight direction and the $\mathrm{X}$ axis is $60^{\circ}$, and the flight speed is $v=400 \mathrm{~m} / \mathrm{s}$.

Assuming the range and cross-range are not sparse, namely all data for translational motion compensation and two-dimensional joint imaging. The reconstruction results shows in Fig. 2.

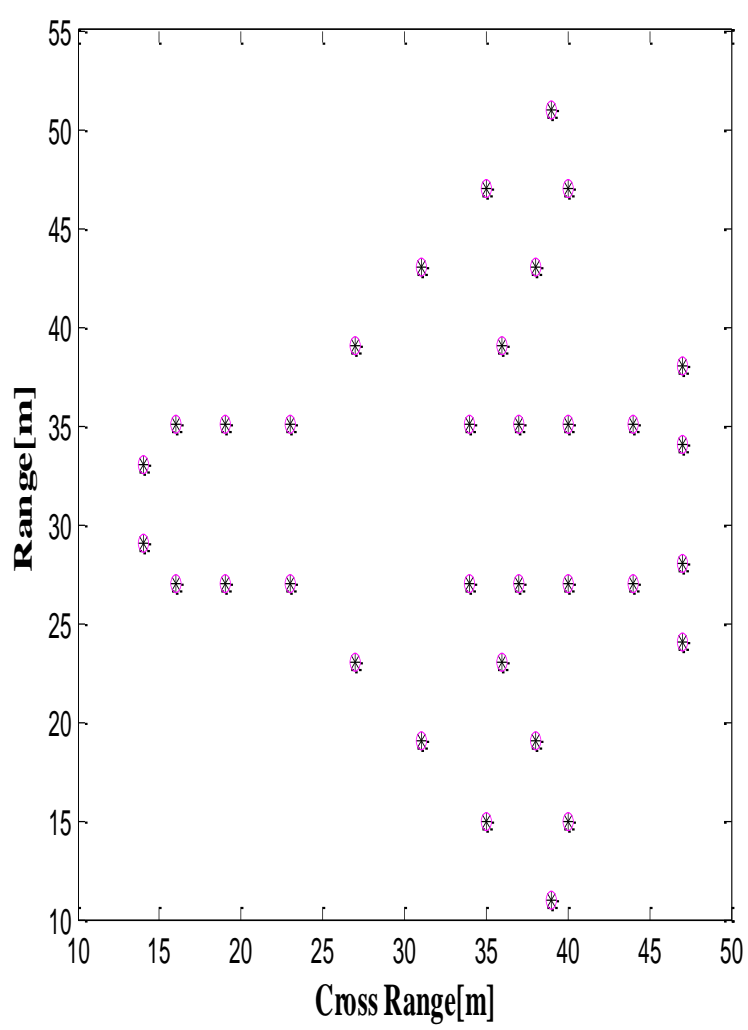

Figure 1. The target model. 


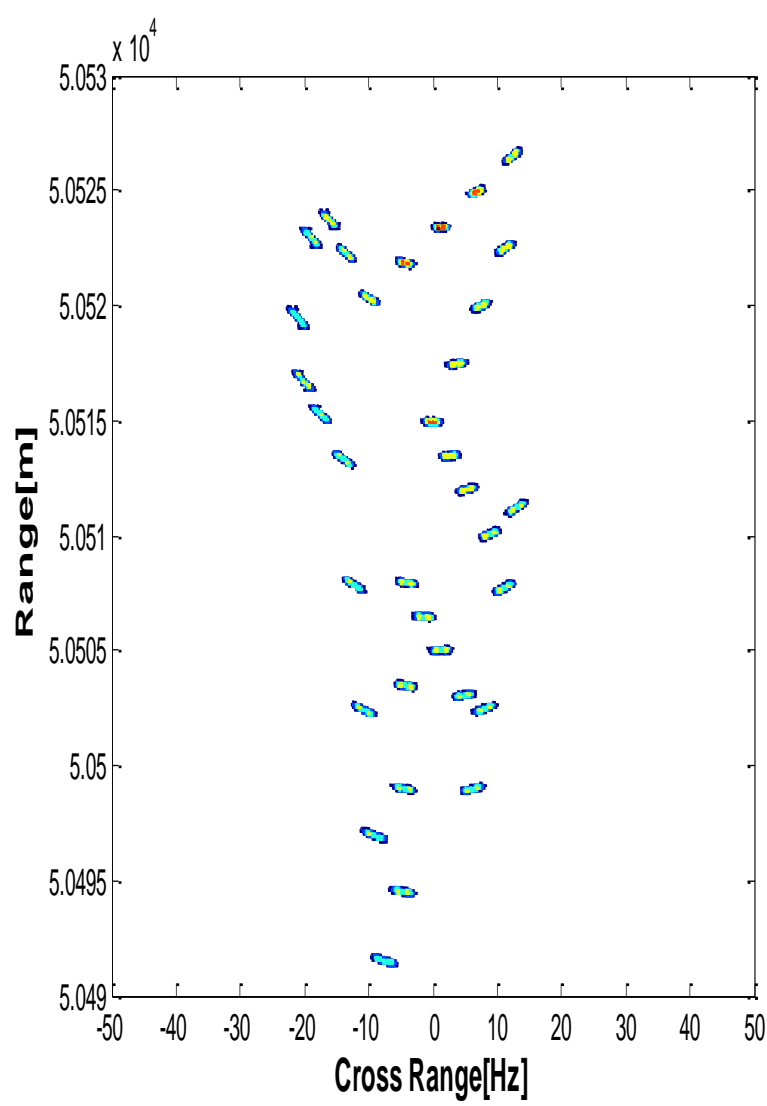

Figure 2. Two dimensional united algorithm.

The simulation results in Fig. 2 can be seen that the imaging effect is better after motion compensation, which proves the validity of this method.

Assuming that other simulation conditions are unchanged, the simulation results are shown in Fig. 3 after motion compensation under different sparse rate conditions. In order to facilitate the performance comparison, the imaging results of the traditional RD algorithm and the two dimensional decoupling method of MSL0 are presented.

In Fig. 3, the first column to the third column is the use of $\mathrm{RD}$ algorithm, two-dimensional decoupling algorithm, the proposed algorithm respectively to get the imaging results. Fig. 3(a)-Fig. 3(c) are different sparse conditions of the imaging results of the three algorithms. It can be seen that: under different sparse conditions, the imaging results of RD algorithm are poor and have more false scattering points. For the two-dimensional decoupling algorithm, with the increase of the sparse rate, the false scattering points of the imaging results are gradually increased, which has a great influence on the imaging results. For the proposed algorithm, with the gradual increase of the sparse rate, the suppression effect of the false scattering point is more ideal, the imaging effect is the most stable. Overall, the proposed algorithm has better imaging effect, and the performance is most stable under different sparse rate conditions.

\section{SUMMARY}

In this paper, we propose a new method based on 2D-FISTA algorithm for the ISAR imaging of sparse frequency stepped frequency waveform, and the simulation results show that the proposed method is effective. The method has fast convergence speed, but it needs to be chosen to achieve a better effect, so how to choose the right parameters to achieve the best results will be the focus of the next research in the future.

\section{REFERENCES}

[1] Ioan Nicolaescu. Improvement of Stepped-Frequency Continuous Wave Ground-Penetrating Radar Cross-Range Resolution, IEEE Transactions on Geoscience and Remote Sensing, 51 (2013) 85-92.

[2] Jun gang Yang, Xiao tao Huang, Tian Jin, et al. Synthetic Aperture Radar Imaging Using Stepped Frequency Waveform, IEEE Transactions on Geoscience and Remote Sensing, 50 (2012) 2026-2036.

[3] Yi min Liu, Hua dong Meng, Gang Li. Velocity Estimation and Range Shift Compensation for High Range Resolution Profiling in Stepped-Frequency Radar, IEEE Geoscience and Remote Sensing Letters, 7 (2010) 791-793.

[4] D. L. Donoho. Compressed sensing, IEEE Trans. Inf. Theory. 52(2006) 1289-1306.

[5] Matthew A. Herman, Thomas Strohmer. High-Resolution Radar via Compressed Sensing, IEEE Transactions on Signal Processing. 57 (2009) 2275-2284

[6] Mariví Tello, Paco López-Dekker, Jordi J. Mallorquí. A Novel Strategy for Radar Imaging Based on Compressive Sensing, IEEE Transactions on Geoscience and Remote Sensing. 48 (2010) 4285-4295.

[7] S. Shah, Y. Yu, A. Petropulu. Step-frequency radar with compressive sampling (SFR-CS), IEEE International Conference on Acoustics, Speech and Signal Processing. (2010) 1686-1689.

[8] Li Jun, Xing Meng dao, Wu Shun jun. Application of Compressed Sensing in Sparse Aperture Imaging of Radar. 978-1-4244-2732-1/09/IEEE, (2009) 651-655.

[9] Jun gang Yang, John Thompson, Xiaotao Huang, et al. Random-Frequency SAR Imaging Based on Compressed Sensing, IEEE Transactions on Geoscience and Remote Sensing. 51(2013) 983-994.

[10] Fu fei Gu, Qun Zhang, Hao Lou, et al.. Two-dimensional sparse synthetic aperture radar imaging method with stepped-frequency waveform, Journal of Applied Remote Sensing. 9 (2015) 1-16.

[11] B. Pang, etal. Imaging enhancement of stepped frequency radar using the sparse reconstru- ction technique, Prog. Electromagn. 140(2013) 63-89.

[12] Ji hong Liu, Shao kunXu, Xun zhang Gao, et al.. Novel imaging methods of stepped frequency radar based on compressed sensing, Journal of Systems Engineering and Electronics, 23(2012) 47-56.

[13] Mohimani H, Babaie-Zadeh M, Gorodnitsy I, et al.. Sparse Recovery using Smoothed LO (SLO): Convergence Analysis DEB/OLD.5073.pdf, (2010).

[14] Fang Y, Wu J J, Huang B M. 2D sparse signal recovery via 2D orthogonal matching pursuit, Science China Information Sciences. 55(2012) 889-897.

[15] Armin E, Massoud B Z, Hamid A M. Two-dimensional random projection, Signal Processing. 91 (2011) 1589-1603.

[16] M.J. Jahromi, M.H. Kahaei. Two-dimensional iterative adaptive approach for sparse matrix solution, Electronics Letters. 50 (2014) 45-47.

[17] Ghaffari A, Babaie-Zadeh M, Jutten C. Sparse decomposition of two dimensional signals[C]. Proc. IEEE Int. Conf Acoustics, Speech and Signal Process. (2009) 3157-3160.

[18] Ghaffari A, Babaie-Zadeh M, Moghaddam H A. Two-dimensional random projection, Signal Process. 91 (2011) 1589-1603. 
[19] Amir B, Marc T. A fast iterative shrinkage-thresholding algorithm for linear inverse problems, SIAM J. Imaging Sciences., 2 (2012) 183-202.
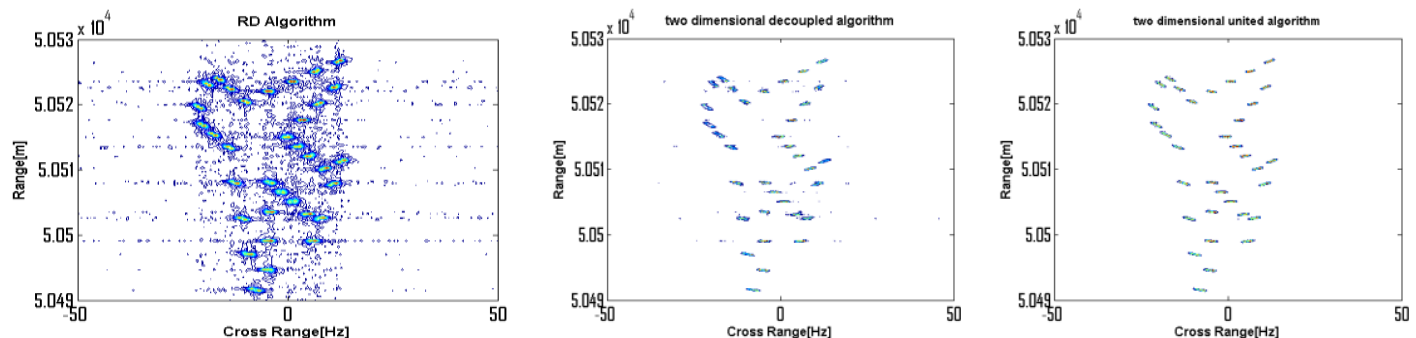

(a) $10 \%$ sparse rate in Range, $10 \%$ sparse rate in Cross Range
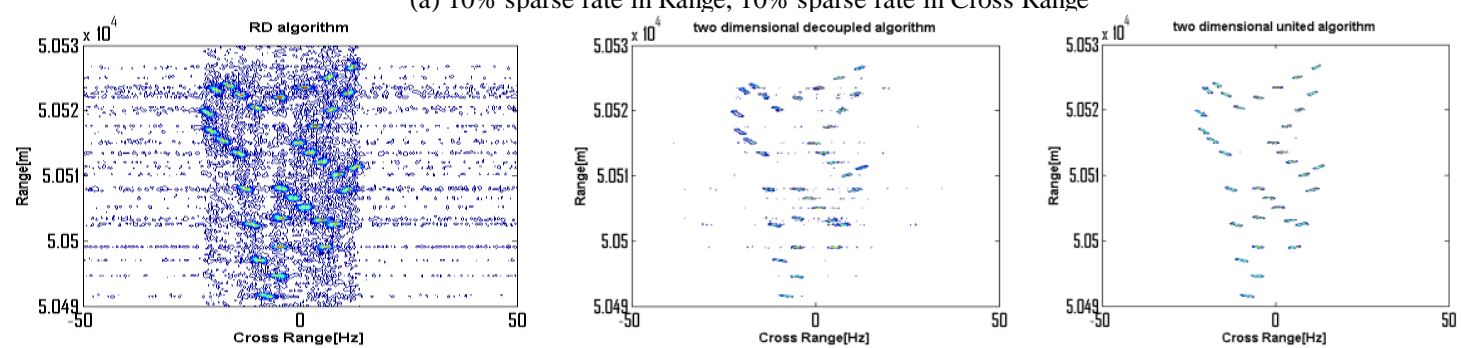

(b) $20 \%$ sparse rate in Range, $20 \%$ sparse rate in Cross Range
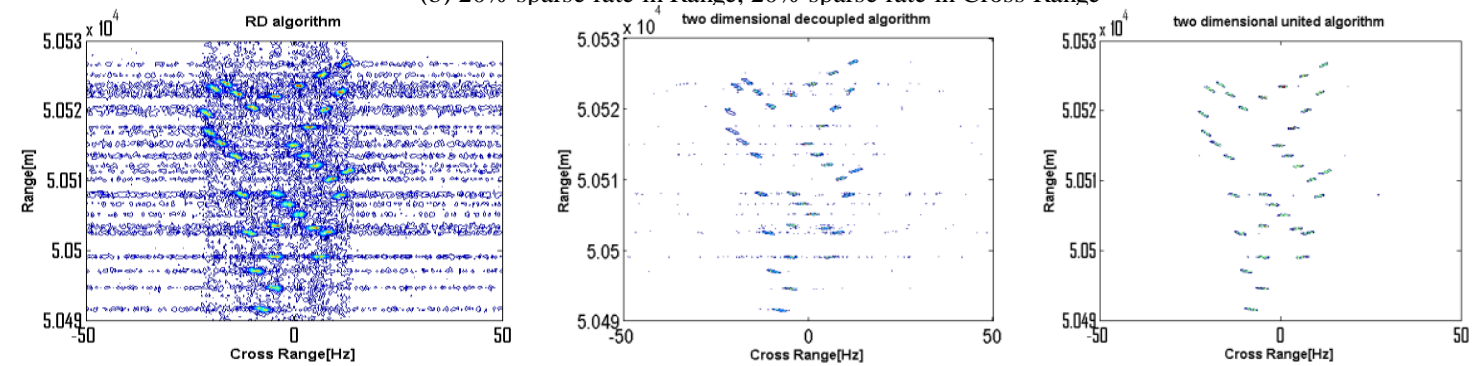

(c) 20\% sparse rate in Range, $40 \%$ sparse rate in Cross Range

Figure 3. Simulation result of the echo of SSFCS signal. 